\title{
CONF- $990651-5$
}

BNL-26114

National Conference on Synchrotron

Bio-3455

Radiation Instrumentation

Gaithersburg, Maryland

June $4-6,1979$

\section{MASTER}

Versatile Spectrometer for Experiments Using Synchrotron Radiation at Wavelengths Greater than $100 \mathrm{~nm}$.

John Clark Sutherland, Edmond J. Desmond and Peter Z. Takacs

Biology Department, Brookhaven National Laboratory Upton, New York 11973 USA

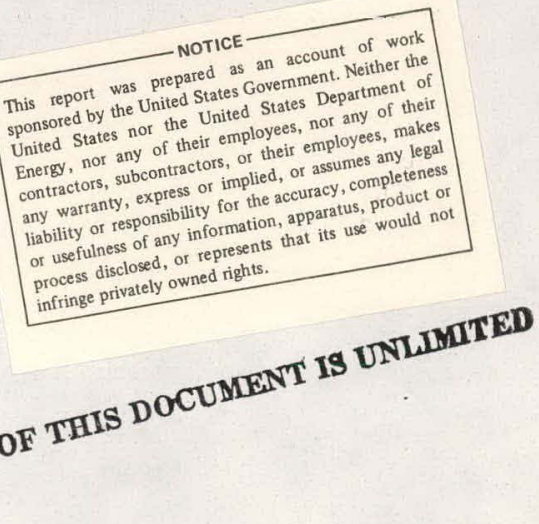

Research supported by the U. S. Department of Energy and by a Research

Career Development Award from National Cancer Institute, US NIH, to JCS. 


\section{DISCLAIMER}

This report was prepared as an account of work sponsored by an agency of the United States Government. Neither the United States Government nor any agency Thereof, nor any of their employees, makes any warranty, express or implied, or assumes any legal liability or responsibility for the accuracy, completeness, or usefulness of any information, apparatus, product, or process disclosed, or represents that its use would not infringe privately owned rights. Reference herein to any specific commercial product, process, or service by trade name, trademark, manufacturer, or otherwise does not necessarily constitute or imply its endorsement, recommendation, or favoring by the United States Government or any agency thereof. The views and opinions of authors expressed herein do not necessarily state or reflect those of the United States Government or any agency thereof. 


\section{DISCLAIMER}

Portions of this document may be illegible in electronic image products. Images are produced from the best available original document. 


\section{INTRODUCTION}

Most experiments using synchrotron radiation ( $S R$ ) have involved the extreme ultraviolet (EUV) and $x$-ray regions of the spectrum, i.e. wavelengths less than about $100 \mathrm{~nm}$. Yet there are certain experiments which can use to advantage the properties of SR at wavelengths greater than $100 \mathrm{~nm}$. To date, measurements of excited state lifetimes have been the most extensively exploited use of $S R$ in this spectral region. There are, however, a number of experiments performed above $100 \mathrm{~nm}$ which are important in biological, chemical and physical spectroscopy and which benefit from other properties of SR, specifically its continuous tunability, intensity, short-term stability, partial polarization and small source size. For a review of the important biophysical experiments in this category, see ref. (1). Prominent among the experiments of this class are: natural and magnetic circular dichroism ( $C D$ and $M C D$ respectively), emission spectroscopies (fluorescence, phosphorescence and related polarization measurements), linear dichroism (induced by electric or magnetic fields, flow, or substrate effects), msec and usec time resolved experiments (stopped flow, temperature jump, etc.) and photoacoustic spectrosçopy (PAS). The impact of SR will, of course, be greatest in those spectral regions where conventional, continuously-tunable sources are least adequate i.e. the UV below $250 \mathrm{~nm}$ and parts of the infrared.

What is the most efficient and economical way to provide instrumentation for these experiments at a SR facility? In a normal laboratory using conventional light sources, the usual approach is to 
provide a separate spectrometer for each class of experiment; one instrument for $C D / M C D$, another instrument for emission studies, another for emission polarization, another for photoacoustic spectroscopy and so on. The limited number of synchrotron ports makes this approach extremely unattractive. An obvious improvement would be to use a common monochromator and have the nature of the experiment be determined by the sample chamber bolted onto its exit slit and the electronics appended thereto.*

*A strong case can be made for dedicating a monochromator, and hence a beam line, to the $\lambda>100$ nm region rather than making do with an EUV monochromator on grounds of flexibility, optical performance, and economy. Biological experiments, for example, are much easier if a window separates the. "wet" sample from the ultrahigh vacuum of the storage ring.

An even better approach is to build an integrated instrument which includes a source monochromator, sample chamber, detectors and a variety of experiment specific components: a magnet for $M C D$, an emission monochromator for fluorescence, and so on. Besides sharing most physical and optical components, it happens that most of the experiments mentioned can be designed so that signal processing is accomplished with a phase-sensitive detector i.e. a "lock-in" amplifier. This type of instrument is ideal for use in a SR facility; 
the time required to change from one operating mode to another is minimized and needless duplication of components is eliminated.**

**The same instrument could also be used for photochemical and photobiological experiments. Fluorescence lifetime measurements, while possible, would require different signal processing electronics.

We have constructed an instrument of the type described which will use wavelengths greater than about $130 \mathrm{~nm}$. Starting sometime in 1979, it will be operated as a CD spectrometer at the SURF II ring at NBS. Other capabilities will be added later. The experiment will be moved to the National Synchrotron Light Source at Brookhaven when that facility begins operation in 1981 .

This synchrotron instrument is conceptually the descendant of an instrument which has operated in our laboratory since 1973 (2) and which has been used to measure $C D$, $M C D$, fluorescence, phosphorescence, linear and circular polarization of emitted light, and fluorescence detected MCD (3). The conventional instrument uses a xenon arc as a light source and non-vacuum monochromators and is thus restricted to wavelengths greater than about $200 \mathrm{~nm}$. In the following sections we shall describe the physical layout of the SUPERB spectrometer, *

*Synchrotron U1traviolet Project for Experimental Research in Biophysics. 
and then discuss in more detail the fully implemented design. The latter section will rely on our conventional instrument which is more fully developed. We will also describe the modular computer system which we have developed to control the conventional spectrometer. 
PHYSICAL CONFIGURATION OF THE SPECTROMETER

A plan view of the SUPERB Spectrometer attached to beam line 6 at SURF II is shown in fig 1. It is sandwiched between the two-meter normal incidence monochromator on beam line 5 (not shown) and the yoke of the storage ring's magnet. All optical components are mounted on an aluminum table which has been shaped to allow access to the source mirror housing, the source monochromator and the sample chamber. The source mirror housing is designed for ultra-high vacuum, the source monochromator for high vacuum and the sample chamber/sample mirror housing for medium vacuum. The three vacuum systems lare separated by transmitting windows.

The source mirror housing contains two spherical mirrors operating near normal incidence to form an image of the tangent point of the electron beam on the plane of the entrance slit of the source monochromator. The sample mirror forms an image of the exit slit of the monochromator at the center of the sample chamber. A polarizer can be placed after the exit slit. The photo-elastic modulator used in $C D$ and other polarization modulation experiments is located between the sample mirror and the sample chamber. The future site of an emission monochromator is also indicated. The sample chamber, modulator housing and sample mirror housing are designed to allow rapid reconfiguration which will become necessary as new experimental capabilities are added. Not shown in fig 1 are the vacuum pumps and the electronic components. 
CONCEPTUAL CONFIGURATION OF THE SPECTROMETER

In fig 2 we show a conceptual configuration of the type of spectrometer we are building. This diagram reflects the configuration of our conventional spectrometer and serves as a goal for development of SUPERB.

In most configurations a modulator is located between the source monochromator and the sample. For polarization experiments (CD, MCD, LD) a photoelastic modulator (PEM) is used $(4,5,6,7,8,9)$. It is preceded by a polarizer.* In emission spectroscopy a mechanical

*At shorter wavelengths it may be preferable to omit the polarizer and utilize the partial polarization inherent in SR. This option is less attractive at longer wavelengths where better polarizers are available and $S R$ is less completely polarized.

chopper is used. For some types of emission measurements it may be feasible to use the time structure of. SR instead of a chopper. In this case it would be possible to obtain lifetime information in addition to spectral distributions although additional electronics will be required.

For biophysical work the sample is usually contained in a aqueous environment; temperature regulation is provided over the range of -20 to $+100 \mathrm{C}$ in the "basic" sample configuration. Auxiliary equipment is available for changing the state of the sample in some useful way such as applying a magnetic field (as in MCD) or reducing the temperature to that of liquid nitrogen or less. 
Light transmitted by the sample impinges on a detector, presently a photomultiplier, where it is converted to an analogue electronic signal. In $C D / M C D / L D$ spectroscopy the a.c. component of the detector's signal is measured with a phase-sensitive detector (PSD) or "lock-in" amplifier. A reference signal for the PSD is provided by the PEM. The "d.c." component of the signal is amplified, and a servo circuit adjusts the sensitivity of the detector to maintain the d.c. component constant as a function of both time and wavelength. Under these conditions the a.c. output signal is proportional to the $C D$ or $L D$ of the sample (10). At present we digitize only the analogue signal from the PSD. In some situations it is desirable to record the output from the d.c. amplifier and the programing signal applied to the detector as shown in Fig. 2.

In PAS the PEM is replaced by a mechanical chopper and the detector is a microphone instead of a light sensitive device (11). After an initial stage of amplification the signal is processed by the PSD referenced to the chopper frequency and phase. The servo loop is inoperative for PAS; the sensitivity of the detector is constant through the experiment. The output of the PSD is converted to digital form and transferred to the computer just as in the case of $C D$ and $L D$ measurements. In a more sophisticated approach; a constant fraction of the incident beam is diverted to a second detector and is used to normalize the output of the PSD to compenstate for changes in source intensity. A similar capability is useful in emission work. 
Part of the energy of the photons absorbed by a sample may reappear, usually at a longer wavelength, in the form of luminescence. Typically, observations are made along an axis perpendicular to the incident beam; other configurations are sometimes advantageous. The spectrum of the luminescence is obtained by scanning an emission monochromator located between the sample and the detector. Alternatively, higher sensitivity can be obtained at the price of spectral resolution by eliminating the emission monochromator. In measurements of the polarization of the emitted radiation a $P E M$ and/or a polarizer may be placed between the sample and the detector as shown.

In emission measurements, the incident beam is mechanically chopped and the signal from the emission detector is analyzed with a PSD. Originally the same analogue PSD was used for both transmission and emission spectroscopies (2). Recently, we have converted the emission channel to photon-counting; in this scheme phase-sensitive detection is accomplished by means of gated scalers. This system, including the associated computer interface, is described elsewhere (12).

A schematic diagram of the computer system is also shown in fig 2. The main processor is a "desk top" class computer (model 4051, "Graphics" system, Tektronix Inc, Beaverton, OR) which contains the microprocessing unit ( $P M U), 32 \mathrm{~K}$ bytes of random-access memory, a storage-type display screen, a keyboard, and a cartridge tape drive. All programing is in the high level BASIC language. The computer's 
operating system and the BASIC interpreter are contained in read-only memory and are thus instantly available when the 40.51 is turned on. A variety of apecial BASIC commands facilitate the display of data in graphical form. Most operator-initiated interactions with the computer are achieved by pressing one of 20 "user definable" keys on the keyboard. The function of each user definable key is determined by the program currently in the memory. For example, to record a spectrum, the operator presses the key designated as "new spectrum". The computer then asks, via the display screen, for a desired starting and ending wavelengths and the wavelength increment at which data is to be collected. These data, as well as information about the nature of the sample are entered via the keyboard and are eventually stored on a flexible disc as is the spectral data. All communications between the computer and the spectrometer are via standardized data exchange protocols. The computer is connected to the emission and polarization spectrometer as well as to a dual flexible disc drive (Tektronix model 4709 ) by a general purpose interface bus (GPIB) which conforms to the specifications of standard 488 of the Institute of Electrical and Electronic Engineers (13). Instruments conforming to this protocol are available from many different manufacturers. In our system the monochromator stepping motors are controlled by an interface which we built; equivalent commercial units have lately become available. Analogue data, for example the signal from the lock-in amplifier which is proportional to circular dichroism, are converted to digital form and transmitted to the 
computer by a Hewlett Packard digital multimeter (model 5300). The photon counting system is interfaced by a module supplied by ORTEC Inc. Once a spectrum is plotted on the display screen of the computer a permanent record can be obtained from a Versatec (Santa Clara, CA) printer-plotter simply by pressing a button on the keyboard of the computer. The computer can access a control host computer via an optional serial (RS-232) interface as shown in fig 2.

This system is very flexible; new components can be obtained from a variety of commercial sources and the system can be easily expanded as requirments develop. In designing the system; greater emphasis was placed on controlling the need for sophisticated programming and custom interfaces than on minimizing initial cost. In comparison to a mini-computer, this system is rather slow. System speed is likely to improve, however, as more of the modules attached to the GPIB acquire their own microprocessors; presently the disc drive and the ORTEC interface contain dedicated MPUs. 


\section{SUMMARY}

The design of the SUPERB spectrometer places great emphasis on flexibility: Optics, electronics and the attendant computer system are of modular design and can be rapidly configured to perform a variety of tasks. This flexibility will permit SUPERB to perform the various experiments described above. Equally important, it should facilitate adaption of SUPERB to perform experiments the nature of which we are presently unaware. 


\section{REFERENCES}

01. J. C. Sutherland: Biophysical Spectroscopy in the visible and ultraviolet using synchrotron radiation, in Biophysical, Biochemical and Biomedical Applications of Synchrotron Radiation, (A. Castellani, ed., Plenum Press, New York, in press).

02. Sutherland, J. C., G. D. Cimino, and J. T. Lowe: Rev. Sci. Instrum. 47 (1976) 358-360.

03. Sutherland, J. C., and H. Low: Proc. Nat. Acad. Sci. USA 72 (1976) $276-280$.

04. Billardon, M., and J. Badoz: C. R. Acad. Sci. 262 (1966) 1672-1675.

Billardon, M., and J. Badoz: C. R. Acad. Sci. 263 (1966) 26-29.

05. Kemp; J. C.: J. Opt. Soc. Am. 59 (1969) 950-954.

06. Mollenauer, L. F., D. Downie, H. Engstrom, and W. B. Grant: App. Opt. 8 (1969) 661-665.

07. Jasperson, J. N., and S. E. Schnatterly: Rev. Sci. Instrum. 40 (1969) $761-767$.

08. Breeze, R. H., and B. Re: Anal. Biochem. 50 (1972) 281-303.

09. Treu, J. I., A. B. Callender, and S. Schnatterly: Rev. Sci. Instrum. 44 (1973) 793-797

10. Velluz, L., M. Legrand, and M. Grossean: Optical Circular Dichroism: Principles, Measurements and Applications (Academic Press, New York, 1965).

11. Rosencwaig, A.: Anal. Chem. 47 (1975) 592A. 
12. Sutherland, J. C., G. D. Cimino, and E. J. Desmond: Anal. Biochem. (in press).

13. IEEE Standard Digital Interface for Programable Instrumentation. (The Institute for Electrical and Electronic Engineers, Inc., New York, 1975). 
FIGURE LEGENDS

Figure 1. Plan view of SUPERB experiment attached to beamline six at SURF II.

Figure' 2. Schematic diagram of the optics, electronics and

computer system of the present configuration of the polarization and emission spectrometer described in Ref. 2. We anticipate that SUPERB will develop along similar lines. 


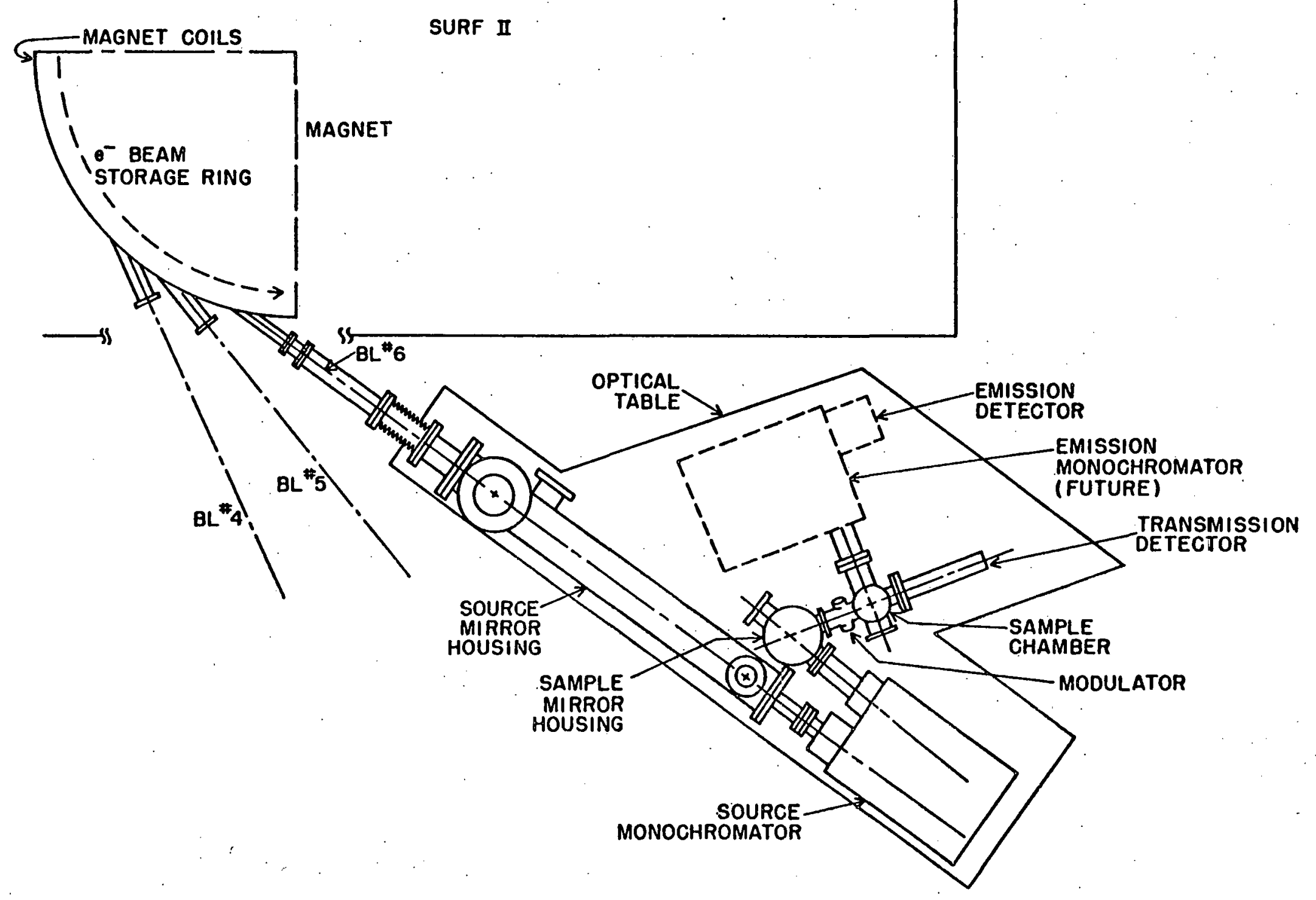




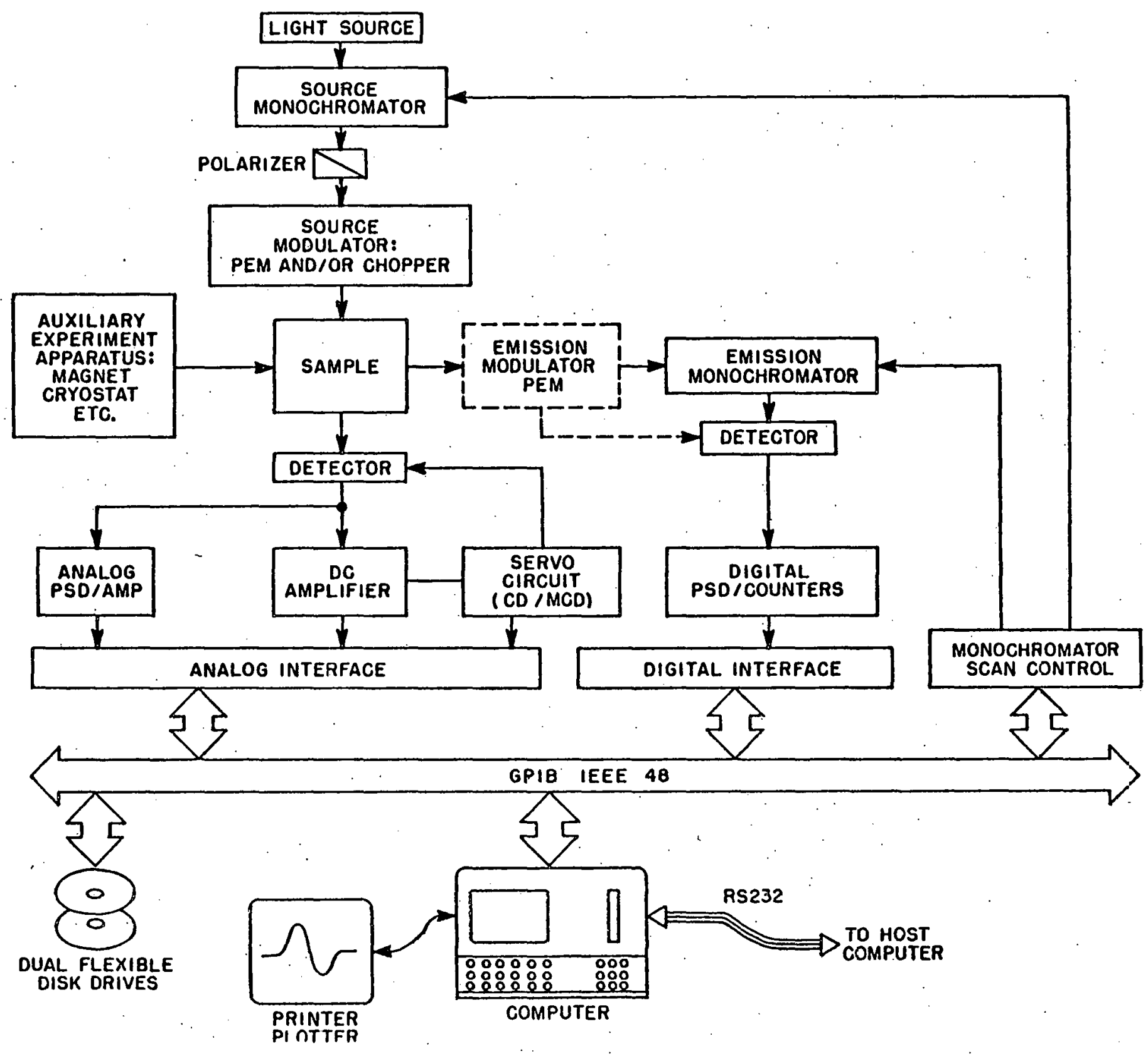

Fig. 2 